“ (C) 2014 IEEE. Personal use of this material is permitted. Permission from IEEE must be obtained for all other uses, in any current or future media, including

reprinting/republishing this material for advertising or promotional purposes, creating new collective works, for resale or redistribution to servers or lists, or reuse of any copyrighted component of this work in other works." 


\title{
Circularly-Polarized Conical-Beam Patch Antenna with Wide Bandwidth and Polarization Diversity
}

\author{
Hang Wong and Wei Lin \\ State Key Laboratory of Millimeter Waves, Department of Electronic Engineering, \\ City University of Hong Kong, \\ Tat Chee Avenue, Kowloon, Hong Kong, \\ Email: weilin6-c@my.cityu.edu.hk
}

\begin{abstract}
This paper introduces a wideband circularly polarized (CP) antenna with polarization diversity for conicalbeam radiation pattern. The antenna composes of a wideband monopolar patch and eight parasitic loop stubs. Shorting pins on the monopolar patch are used to obtain a wide impedance bandwidth. And the other set of shorting pins on the loop stubs are utilized for realizing a wide axial ratio bandwidth. PIN diodes are applied to achieve polarization diversity. Right handed circular polarization (RHCP) and left handed circular polarization (LHCP) can be altered by controlling the operation of different group of diodes. Obtained results show that the proposed antenna has the wide impedance bandwidth of $29 \%$ and the wide axial ratio bandwidth of $15 \%$ for both $C P$ modes. In addition, the stable conical-beam radiation patterns can be found across the operating bandwidth with the maximum gain of $4.4 \mathrm{dBic}$. This antenna is suitable to be mounted on moving vehicles for geostationary satellite communication without satellite tracking.
\end{abstract}

Index Terms - Circular polarization, conical-beam pattern, polarization diversity, wide bandwidth, patch antenna.

\section{INTRODUCTION}

Satellite communication services have been developing rapidly during the past decades. Expect for navigation satellite systems like Compass Navigation Satellite System (CNSS) of China and Global Positioning System (GPS) from the United State, geostationary satellite systems can provide many services like TV broadcasting, satellite radio, satellite phone and other special communications. Meanwhile, antennas play an important role in the satellite systems. For the navigation satellite system, it requires the antenna with a broadside radiation pattern of wide beamwidth, such that it can communicate with the satellite at the low elevation angle. But for the geostationary satellite application, the antenna with a conical-beam pattern is desired as it provides a better capability in the satellite tracking. Furthermore, the antenna with polarization diversity has become more and more popular due to its contribution to the enhancement of signal sensitivity. .

Many efforts have been investigated in $\mathrm{CP}$ antennas with polarization diversity [1-6]. Reported works in [1-3] applied PIN diodes on microstrip patch antennas to alter the structure of radiating elements on patches for polarization diversity. In addition, other works in

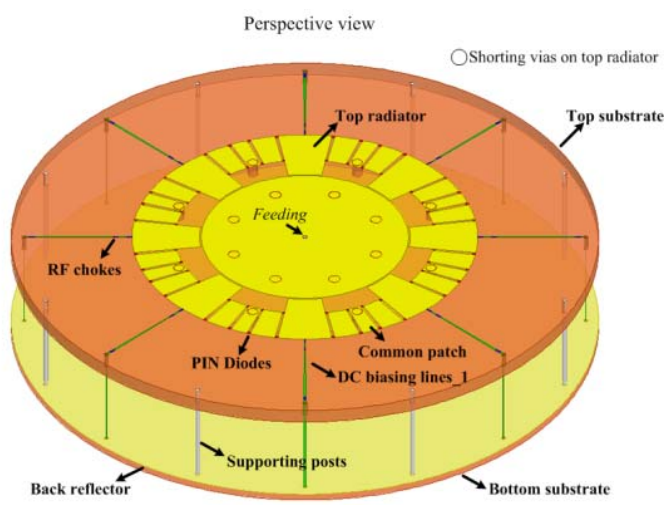

Fig. 1. Antenna geometry of CP conical-beam antenna with polarization reconfigurable.

[4-5] placed RF switches on the feeding network of the antenna to control different operating modes for polarization diversity. All above designs can be used in a navigation satellite system due to their broadside radiation patterns. For geostationary satellite applications, one design in [6] can achieve conical-beam radiation pattern with polarization diversity. However, both impedance and axial ratio bandwidths are very narrow ( $<4.3 \%$ and $<2 \%$ respectively).

In this paper, we propose a wideband CP conical-beam antenna with polarization diversity. Wideband characteristics are obtained by applying shoring pins on a monopolar patch and loop stubs. Furthermore, PIN diodes are utilized to alter two orientations of the loop stubs for generating either RHCP or LHCP wave propagation.

\section{ANTENNA STRUCTURE}

The proposed antenna composes of a top radiator, a back reflector and DC biasing lines as shown in Fig. 1. The top radiator is a center-fed monopolar patch surrounded by eight coupling loop stubs. Eight sets of shorting pins are placed on both monopolar patch and loop stubs. PIN diodes are connected between the patches and the coupling loop stubs. The DC lines are connected to the big patches of the top radiator. In addition, a reflector is located below with other parts of DC lines fabricated on both layers of the bottom substrate. The antenna is fed by a coaxial cable and supported by some plastic posts. 


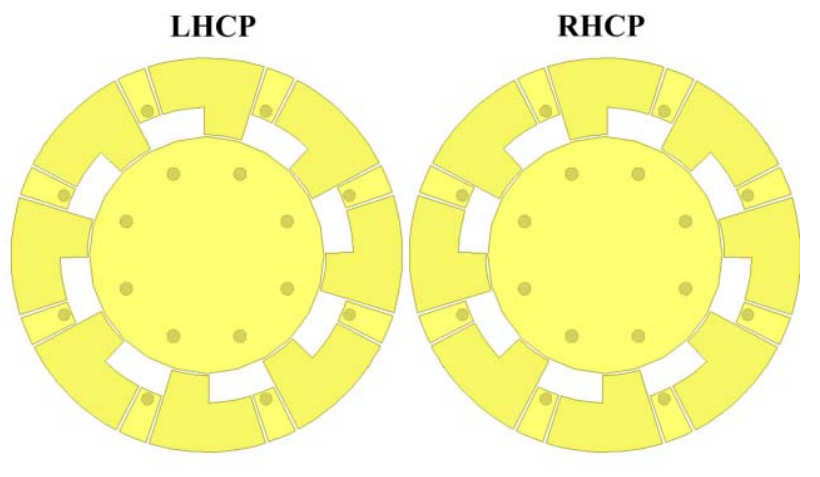

Fig. 2. Polarization diversity realization.

\section{OPERATING PRINCIPLE}

We use PIN diodes to realize polarization diversity as seen in Fig. 2. When the four diodes at the left side of the common patch are turned on, the orientation of the radiating loop stubs will be anti-closewise for LHCP mode. On the other hand, antenna will radiate RHCP waves if the diodes at the right side are in operation. Therefore, polarization diversity is realized by choosing the proper PIN diodes that can be operating in our targeted frequency band. In addition, wideband characteristics are realized by introducing shorting pins on monopolar patch and loop stubs as seen in Fig. 3.

\section{MeAsured Results}

The fabricated antenna was measured with SATIMO measurement system. Fig. 4 presents the measured impedance bandwidths and the antenna gain. The antenna has the wide impedance bandwidths of $29 \%$ and the maximum gain of $4.4 \mathrm{dBic}$. In addition, it also obtains the wide AR bandwidth of $15 \%$ as shown in Fig. 5 which covers the downlink of standard $\mathrm{C}$ band (3.625 to $4.2 \mathrm{GHz}$ ) for geostationary satellite communications. Finally, the conicalbeam patterns for both $\mathrm{CP}$ modes are achieved as seen in Fig. 6.

\section{ACKNOWLEDGMENT}

This project was supported in part by the Research Grants Council of the Hong Kong SAR, China (Project No. CityU 138413).

\section{REFERENCES}

[1] Y. J. Sung, T. U. Jang, and Y.-S. Kim, "A reconfigurable microstrip antenna for switchable polarization," IEEE Microwave and Wireless Components Letters, vol. 14, No. 11, pp. 534-536, 2004

[2] Y. J. Sung, "Reconfigurable patch antenna for polarization diversity," IEEE Trans. Antennas Propag., vol. 56, no. 9, pp. 2053-2054, Sep., 2008.

[3] T. Song, Y. Lee, D. Ga and J. Choi, "A Polarization Reconfigurable Microstrip Patch Antenna using PIN Diodes," Proceedings of APMC 2012, Dec. 4-7, 2012.

[4] J. S. Row, W. L. Liu, and T. R. Chen, "Circular polarization and polarization reconfigurable designs for annular slot antennas," IEEE Trans. Antennas Propag., vol. 60, no.12, pp. 5998-6002, Dec. 2012.

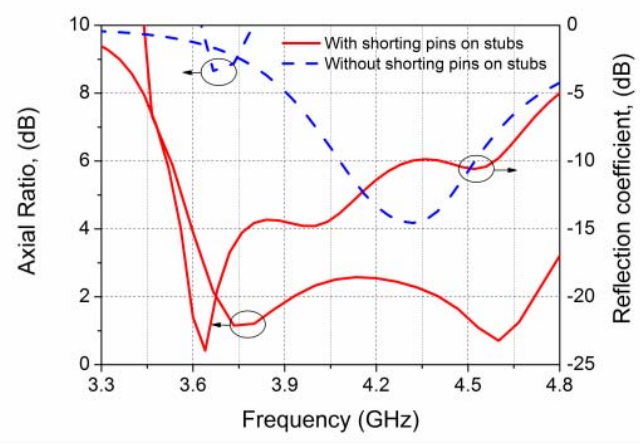

Fig. 3. Impedance and AR bandwidths comparison between antenna with and without shorting pins on coupling loop stubs.

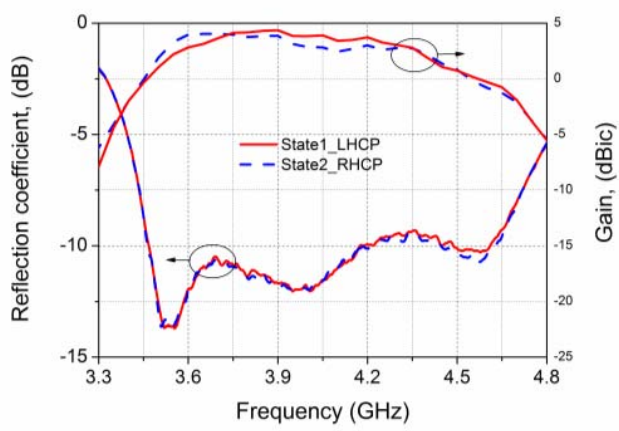

Fig. 4. Measured impedance bandwidth and gain.

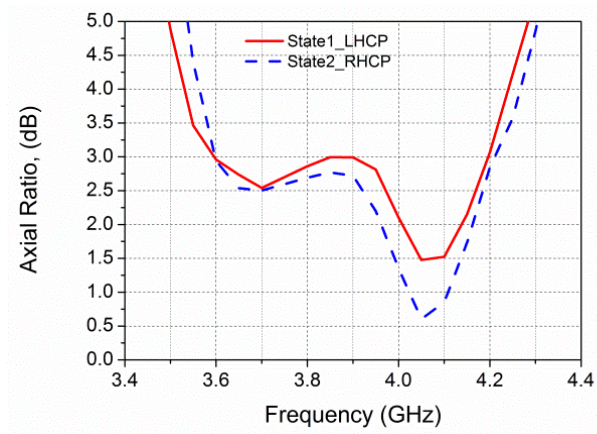

Fig. 5. Measured AR bandwidth.

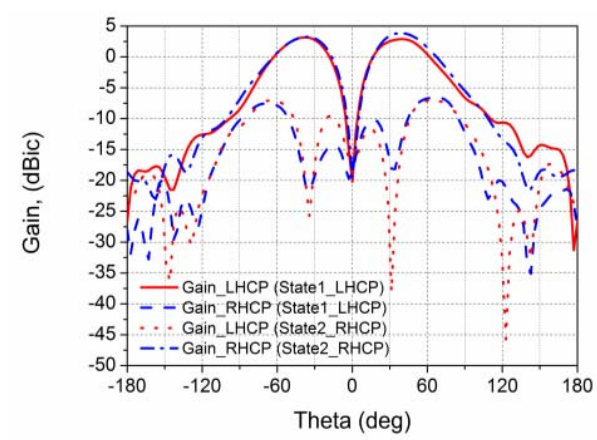

Fig. 6. Measured radiation patterns

[5] Y. Li, Z. J. Zhang, W. H. Chen and Z. H. Feng, "Polarization reconfigurable slot antenna with a novel compact CPW-to-slotline transition for WLAN application," IEEE Antenna and wireless propag. letters, vol. 9, pp. 252-255, 2010.

[6] J. S. Row and M. C. Chan, "Reconfigurable circularly-polarized patch antenna with conical beam," IEEE Trans. Antennas Propag., vol. 58 , no.8, pp. 2753-2757, Aug., 2010. 